\title{
Machine learning corrected quantum dynamics calculations
}

\author{
A. Jasinski $\odot$, J. Montaner $\odot$, and R. C. Forrey $\odot^{*}$ \\ Department of Physics, Penn State University, Berks Campus, Reading, Pennsylvania 19610-6009, USA \\ B. H. Yang $\odot$ and P. C. Stancil \\ Department of Physics and Astronomy and the Center for Simulational Physics, University of Georgia, Athens, Georgia 30602, USA \\ N. Balakrishnan $\odot$ \\ Department of Chemistry and Biochemistry, University of Nevada, Las Vegas, Nevada 89154, USA

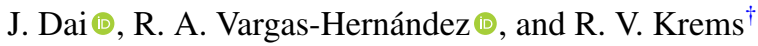 \\ Department of Chemistry, University of British Columbia, Vancouver, British Columbia, Canada V6T 1Z1
}

(Received 10 January 2020; accepted 10 August 2020; published 27 August 2020)

\begin{abstract}
Quantum scattering calculations for all but low-dimensional systems at low energies must rely on approximations. All approximations introduce errors. The impact of these errors is often difficult to assess because they depend on the Hamiltonian parameters and the particular observable under study. Here, we illustrate a general, system- and approximation-independent, approach to improve the accuracy of quantum dynamics approximations. The method is based on a Bayesian machine learning (BML) algorithm that is trained by a small number of exact results and a large number of approximate calculations, resulting in ML models that can generalize exact quantum results to different dynamical processes. Thus, a ML model trained by a combination of approximate and rigorous results for a certain inelastic transition can make accurate predictions for different transitions without rigorous calculations. This opens the possibility of improving the accuracy of approximate calculations for quantum transitions that are out of reach of exact scattering theory.
\end{abstract}

DOI: 10.1103/PhysRevResearch.2.032051

Introduction. Quantum dynamics problems with timeindependent Hamiltonians are often solved by representing the Hamiltonian eigenstates by a basis set expansion and numerically integrating the resulting set of coupled equations [1-3]. As the complexity of quantum systems increases, the number of coupled equations required to obtain accurate solutions becomes prohibitively large. Therefore, quantum dynamics calculations are often based on decoupling approximations that reduce the problem to smaller, independent sets of coupled equations. These approximations necessarily introduce errors into the dynamical results. The impact of these errors is often difficult to assess because they depend on the Hamiltonian parameters and the particular observable under study. In this Rapid Communication, we demonstrate a general, system- and approximation-independent, method to enhance the accuracy of the approximations. The approach is based on Bayesian machine learning (BML) models that learn correlations between the approximate and rigorous results

\footnotetext{
*rcf6@psu.edu

†rkrems@chem.ubc.ca
}

Published by the American Physical Society under the terms of the Creative Commons Attribution 4.0 International license. Further distribution of this work must maintain attribution to the author(s) and the published article's title, journal citation, and DOI. for some dynamical processes and transfer this information to correct the quantum dynamics approximations for other processes.

We consider the inelastic scattering problem of two diatomic molecules prepared in a wide range of internal quantum states and undergoing collisions over a wide range of collision energies. Accurate predictions of probabilities for such collisions are required for applications in astrophysics [4], planetary atmosphere models, the development of new crossed-beam experiments for precision measurements [5], cold chemistry $[6,7]$, as well as the mechanistic understanding of microscopic collision dynamics [8,9]. Rigorous quantum calculations of inelastic molecule-molecule scattering must be performed in six nuclear dimensions and account for all couplings between the internal and translational motion states of the molecules. The complexity of the problem can be reduced by eliminating some of the angular momentum couplings and/or freezing some degrees of freedom. We consider an approximation that reduces the active configuration space to five dimensions (5D) and neglects angular momentum couplings giving rise to Coriolis interactions. We focus on state-resolved inelastic transitions and demonstrate that ML models trained by a combination of approximate and (a small number of) rigorous results for some transitions can make accurate predictions for different transitions without rigorous calculations, with accuracy exceeding that of the approximate calculations. 
Machine learning has been previously combined with quantum calculations in order to solve problems in quantum condensed-matter physics [10-12], quantum chemistry [13-19], and molecular dynamics [20,21]. These studies can be classified into approaches based on artificial neutral networks (NNs) and kernel-based methods, including Bayesian ML using Gaussian processes (GPs) [22]. NNs generally require a large number of input data to produce accurate models. In the present Rapid Communication, our goal is to build models of quantum dynamical observables improved by a small number of rigorous calculations. Previous applications show that Bayesian ML based on GPs can produce powerful, nonparametric prediction models based on very sparse data [23-28]. This makes GPs ideally well suited for Bayesian model calibration (BMC) [28,29], which aims to compensate for the (unknown) deficiencies of a simulation model in a flexible, nonparametric way. In the context of quantum dynamics, BMC has been used to interpolate quantum results by a ML model trained with a large number of classical trajectory calculations [30] and to improve the predictions of transition state theory for bimolecular chemical reactions [31]. $\mathrm{BMC}$ has also been used in quantum chemistry to enhance the accuracy of potential energy calculations [32].

We consider state-resolved inelastic collisions between two diatomic molecules in well-defined quantum states specified by the vibrational $(v)$ and rotational $(j)$ quantum numbers

$$
A\left(v_{1}, j_{1}\right)+B\left(v_{2}, j_{2}\right) \rightarrow A\left(v_{1}^{\prime}, j_{1}^{\prime}\right)+B\left(v_{2}^{\prime}, j_{2}^{\prime}\right),
$$

where the subscripts label the different molecules. Our goal is to build a ML model of cross sections for such collisions. We impose the following requirements on the model: The model must be easy to evaluate; the model must be more accurate than the results of the approximate dynamical calculations; the model must be nonparametric to adapt to increasing information about the scattering process, which may reveal new dynamical features, such as resonances; and the model must require as few rigorous quantum calculations as possible.

Our ML models are based on GPs so we begin by a brief description of a GP (see Refs. [22,28] for more details). The purpose of a GP model is to make a prediction of some quantity $y$ at an arbitrary point $\boldsymbol{x}$ of a $\mathcal{D}$-dimensional space, given a finite number $n$ of values $\boldsymbol{y}=\left(y_{1}, \ldots, y_{n}\right)^{\top}$, where $y_{i}$ is the value of $y$ at $\boldsymbol{x}_{i}$. In the absence of noise in $y_{i}$, the goal is to infer the function $f(\boldsymbol{x})$ that interpolates $y_{i} \Leftarrow f\left(x_{i}\right)$. GPs infer the conditional distribution over functions $p(f \mid \boldsymbol{y})$. The conditional mean of such distribution as a function of $\boldsymbol{x}_{*}$ is given by [22]

$$
\mu\left(\boldsymbol{x}_{*}\right)=K\left(\boldsymbol{x}_{*}, \boldsymbol{x}\right)^{\top}\left[K(\boldsymbol{x}, \boldsymbol{x})+\sigma_{n}^{2} I\right]^{-1} \boldsymbol{y},
$$

where $\boldsymbol{x}_{*}$ is a point in the input space where the prediction is to be made, and $K(\boldsymbol{x}, \boldsymbol{x})$ is the $n \times n$ square matrix with the elements $K_{i, j}=k\left(\boldsymbol{x}_{i}, \boldsymbol{x}_{j}\right)$. The function $k\left(\boldsymbol{x}^{\prime}, \boldsymbol{x}^{\prime \prime}\right)$ represents the covariance between the normal distributions of $y$ at $\boldsymbol{x}^{\prime}$ and $\boldsymbol{x}^{\prime \prime}$

The unknown parameters of this function are found by maximizing the log marginal likelihood function,

$$
\log p(\boldsymbol{y} \mid \boldsymbol{\theta})=-\frac{1}{2} \boldsymbol{y}^{\top} K^{-1} \boldsymbol{y}-\frac{1}{2} \log |K|-\frac{n}{2} \log (2 \pi),
$$

where $\boldsymbol{\theta}$ denotes collectively the parameters of $k$ and $|K|$ is the determinant of the matrix $K$. Given the $k$ functions thus found, Eq. (2) is a GP model.

Here, we propose to use two types of ML models based on GPs. First, we define the ML model of a quantum dynamics cross section as [29,30]

$$
\sigma_{\mathrm{ML}}(\boldsymbol{x})=a \mathcal{F}(\boldsymbol{x})+\mathcal{G}(\boldsymbol{x}),
$$

where $\mathcal{F}$ and $\mathcal{G}$ are independent GPs and $a$ is a variable parameter. Here, $\mathcal{F}$ is designed to describe the $\boldsymbol{x}$ dependence of approximate dynamical results and $\mathcal{G}(\boldsymbol{x})$ infers the difference between the approximate and accurate calculations. This is equivalent to $\Delta$-learning algorithms that have been used for quantum chemistry applications [33].

Second, we generalize the GP to a multioutput GP F $(x) \Rightarrow$ $\left[\mathcal{F}_{1}(\boldsymbol{x}), \mathcal{F}_{2}(\boldsymbol{x})\right]$, with two outputs corresponding to the approximate and exact results [34]. The covariance matrix $K$ for such a GP includes correlations between inputs $\boldsymbol{x}$ and $\boldsymbol{x}^{\prime}$, as well as correlations between the outputs. The ML model of accurate cross sections is given by

$$
\sigma_{\mathrm{ML}}(\boldsymbol{x})=\mathcal{F}_{2}(\boldsymbol{x}) .
$$

Models (4) and (5) learn the correlations between the accurate and approximate results in different ways. We will illustrate different consequences of these two approaches.

To select the covariance functions $k\left(\boldsymbol{x}, \boldsymbol{x}^{\prime}\right)$ for the above models, we use the algorithm from Refs. [23-25], which builds up the covariance complexity using the Bayesian information criterion. We find that models (4) perform accurately already with a simple Matérn function of Ref. [25], while models (5) require a more complex covariance represented by a linear combination of a product of Matérn functions and a radial basis function, as defined in Ref. [25]. The specific details about the models are presented in the Supplemental Material [35].

To build a general ML model $\sigma_{\mathrm{ML}}$, we define the input variable space $\boldsymbol{x}$ as follows,

$$
\boldsymbol{x}=\left\{E_{c}, \Delta E_{\text {int }}, \Delta A_{\text {int }}, \Delta v_{1}, \Delta j_{1}, \Delta v_{2}, \Delta j_{2}\right\},
$$

where $E_{c}$ is the collision energy, $\Delta E_{\text {int }}$ is the change of the internal energy of the molecules, $\Delta A_{\text {int }}$ is the change of the angular momentum of the collision complex, $\Delta v=$ $v^{\prime}-v$, and $\Delta j=j^{\prime}-j$. This allows our models to classify transitions by the corresponding quantum number gaps and make predictions about cross sections for specific transitions based on information about other transitions. For example, we will illustrate that rigorous results for the $v=1, j=0 \rightarrow$ $v=0, j=2$ transition could be used to make predictions of cross sections for the $v=2, j=0 \rightarrow v=1, j=2$ transition, without rigorous calculations.

We consider collisions of $\mathrm{SiO}$ and $\mathrm{CO}$ with para- $\mathrm{H}_{2}$. Details of the potential energy surfaces and scattering calculations have been reported previously [36-40]. For each system, we perform six-dimensional close-coupling (6DCC) and five-dimensional coupled-state (5DCS) calculations. We use 6DCC and 5DCS calculations as "rigorous" and "approximate" results, respectively. To illustrate the generality of our approach, we also modulate 5DCS calculations by a random sinusoidal function and treat the resulting data as 
"approximate" cross sections for training ML models (see more details below).

Cross sections at a given collision energy $E_{c}$ are calculated using the appropriate $T$ matrix for the respective $6 \mathrm{DCC}$ and 5DCS formulations. For the 6DCC calculations, the $T$ matrix is diagonal with respect to the total angular momentum quantum number $J$, defined by the vector relations $\vec{J}=\vec{l}+\vec{j}_{12}$ and $\vec{j}_{12}=\vec{j}_{1}+\vec{j}_{2}$, where $\vec{l}$ is the orbital angular momentum. For the 5DCS calculations, the $T$ matrix is independent of $J$ and diagonal with respect to $m_{1}$ and $m_{2}$, the projection quantum numbers of $\vec{j}_{1}$ and $\vec{j}_{2}$. For the CS approximation, $\vec{l} \equiv J$ is the average value of $l$ between $\left|J-j_{12}\right|$ and $J+j_{12}$. For the internal energy of the molecules, we use

$$
\begin{gathered}
E_{\mathrm{int}}^{(i)}=w_{e}^{(i)}\left(v_{i}+1 / 2\right)-w_{e}^{(i)} x_{e}^{(i)}\left(v_{i}+1 / 2\right)^{2}+B_{v_{i}} j_{i}\left(j_{i}+1\right), \\
A_{\mathrm{int}}^{(i)}=B_{v_{i}}\left(2 j_{i}+1\right) \\
B_{v_{i}}=B_{e}^{(i)}-a_{e}^{(i)}\left(v_{i}+1 / 2\right)
\end{gathered}
$$

with coefficients given in Ref. [41].

We quantify the accuracy of our results by root-meansquared (rms) relative error

$$
\text { rms relative error }=\sqrt{\frac{1}{N} \sum_{f}^{N}\left(\frac{\sigma_{i \rightarrow f}^{\text {exact }}-\sigma_{i \rightarrow f}^{\text {approx }}}{\sigma_{i \rightarrow f}^{\text {exact }}}\right)^{2}},
$$

where $\sigma^{\text {exact }}$ is the 6DCC result, $\sigma^{\text {approx }}$ corresponds to either the 5DCS value or the ML prediction, and $i / f$ denote the initial/final states.

Results. We begin by illustrating that ML can be used to improve approximate results for rovibrational transitions, for which accurate calculations are computationally challenging. To do this, we consider a set of 21 transitions

$$
\begin{aligned}
\left(v_{1}, j_{1}, v_{2}, j_{2}\right) & =(1,0,0,0) \longrightarrow\left(v_{1}^{\prime}, j_{1}^{\prime}, v_{2}^{\prime}, j_{2}^{\prime}\right) \\
& =(0, X, 0,0),
\end{aligned}
$$

where $X=0-20$. For each of these transitions, we calculate the 6DCC and 5DCS cross sections in the energy interval between 1 and $1000 \mathrm{~cm}^{-1}$. Our goal is then to predict the cross sections for different transitions,

$$
(2,0,0,0) \rightarrow(1, X, 0,0),
$$

without any further 6DCC calculations. We train our model (4) by a combination of 6DCC and 5DCS cross sections for the transitions (11) and 5DCS results for the transitions (12). The model is then used to predict the cross sections for the transitions (12). Figure 1 illustrates that the accuracy of the ML predictions is $10 \%-30 \%$ better than of the approximate results.

The trend observed in Fig. 1 is general. To illustrate this, we consider transitions involving state changes of both molecules and a different system. We use 6DCC to compute the cross sections for the rovibrational transitions from the initial state $\left(v_{1}, j_{1}, v_{2}, j_{2}\right)=(1,4,0,0)$ for $\operatorname{SiO}\left(v_{1}, j_{1}\right)+$ $\mathrm{H}_{2}\left(v_{2}, j_{2}\right)$ collisions. These cross sections are then used to predict state-resolved cross sections for the $(1,5,0,0)$ initial state making transitions with nonzero values of $\Delta v_{1}, \Delta j_{1}$, and

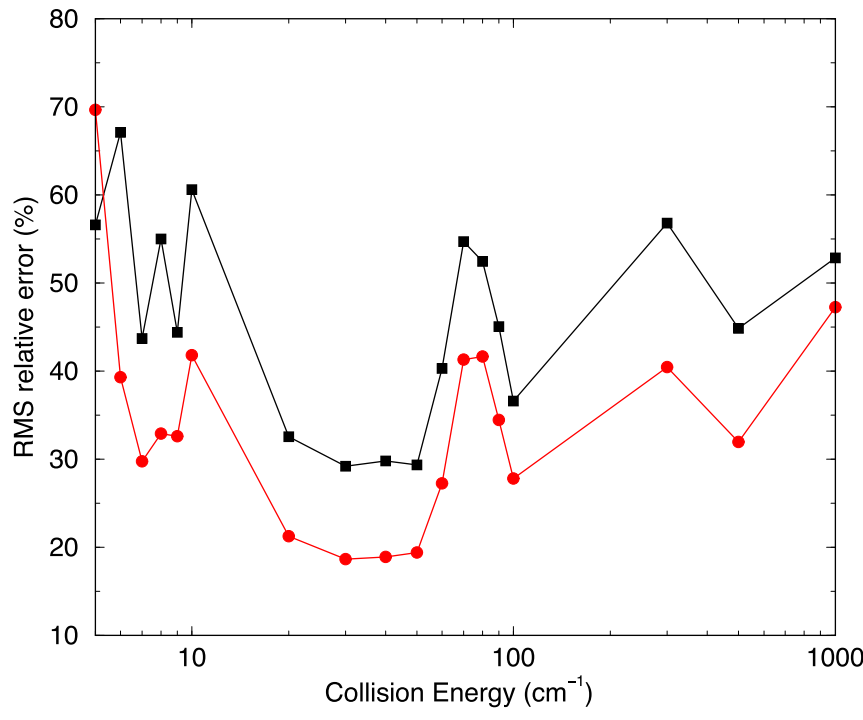

FIG. 1. The rms relative error for rovibrational deexcitation of $\mathrm{CO}$ due to collision with $\mathrm{H}_{2}$ : squares, 5DCS; circles, ML results. The ML model (4) is trained using the 5DCS and 6DCC results for 21 transitions (11) and only 5DCS for 21 transitions (12). No 6DCC results for transitions (12) are used to train the model (4). The error depicted is for 21 transitions (12).

$\Delta j_{2}$. Errors are shown in Fig. 2 for $\Delta v_{1}=-1$ and $\Delta j_{2}=2$. The upper panel shows the errors calculated for all 21 transitions to the final states $(0, X=[0,20], 0,2)$, whereas the lower panel illustrates the reduction of the error for ten transitions with the most significant ML improvement. We note that the accuracy of the 5DCS calculations used for training ML models in Figs. 1 and 2 is poor and that none of the ML predictions in these figures used 6DCC computations for the transitions shown. The accuracy of the ML predictions does not depend on the accuracy of the approximation. Instead, it is generally determined by correlations between the rigorous and approximate results for the different transitions. When these correlations are strong, the ML predictions can give very accurate results. We illustrate this next by considering pure rotational transitions (Fig. 3).

Figure 3 compares the performances of the ML models (4) and (5) for predictions of the cross sections for pure rotational relaxation of $\mathrm{SiO}$ molecules initially in state $\left(v_{1}=1, j_{1}=5\right)$. The ML models are built using the 6DCC cross sections for rotational relaxation of $\operatorname{SiO}\left(v_{1}=0, j_{1}=5\right)$. These models are then used to correct the 5DCS results for the relaxation from the state $\left(v_{1}=1, j_{1}=5\right)$. As can be seen, the ML corrected results are in perfect agreement with the rigorous $6 \mathrm{DCC}$ calculations, used here for testing purposes only. Note that the CC calculations for the vibrationally ground state extend only to the collision energy $10^{3} \mathrm{~cm}^{-1}$. Figure 3 illustrates that at energies above $10^{3} \mathrm{~cm}^{-1}$, the ML model (4) recovers the CS results, while the ML model (5) extrapolates the 6DCC results.

To illustrate that the ML predictions do not rely on the accuracy of the coupled-state calculations, we modulate the 5DCS results for the $j=5 \rightarrow j^{\prime}=0$ transition in Fig. 3 by a random sinusoidal function. This leads to the dotted curve shown in the upper panel of Fig. 3. The symbols for the 

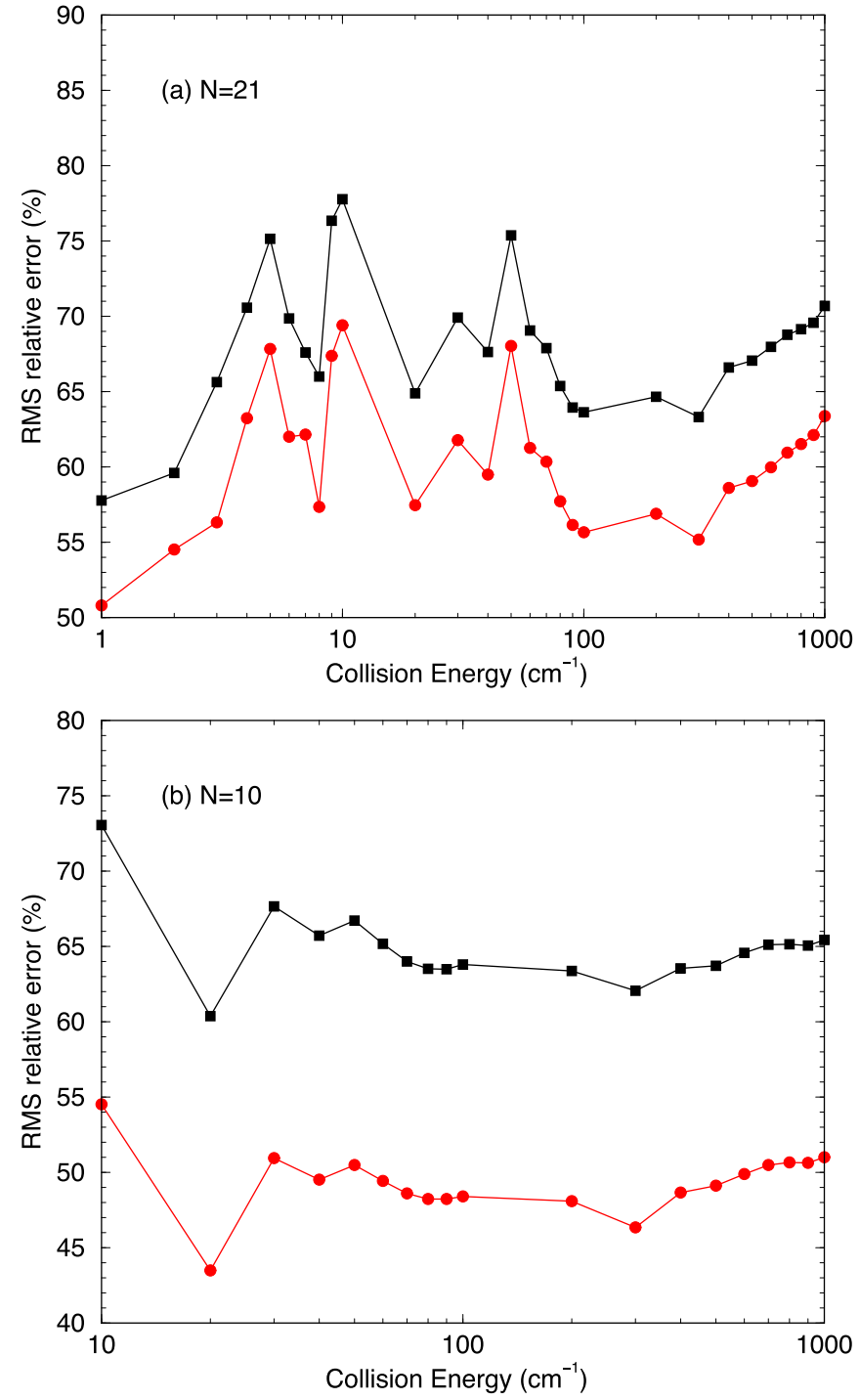

FIG. 2. The rms relative errors for rovibrational deexcitation of $\mathrm{SiO}$ and simultaneous rotational excitation of $\mathrm{H}_{2}$. The curves correspond to transitions from the initial state $\left(v_{1}, j_{1}, v_{2}, j_{2}\right)=(1,5,0,0)$ to the final states $(0, X, 0,2)$, where $X=0-20$. The black curves connecting the squares are the errors of the 5DCS results, and the red curves connecting the circles are the errors of the ML results. The ML models (4) are trained by the cross sections for the transitions $(1,4,0,0) \rightarrow(0, X, 0,2)$, where $X=0-20$. The lower panel illustrates the error reduction for ten transitions with the most significant ML improvement.

$j=5 \rightarrow j^{\prime}=0$ transition in Fig. 3 (upper panel) are the ML predictions using the dotted curve instead of the 5DCS results as the training data. We emphasize again that solid curves in Figure 3 have not been used for training the ML models.

In summary, we have illustrated a general, system- and approximation-independent, approach to improve the accuracy of quantum dynamics approximations. The method is based on GP models that infer correlations between approximate and rigorous results for some transitions and transfer the learned information to other transitions. We have shown that these correlations can be learned either by using an
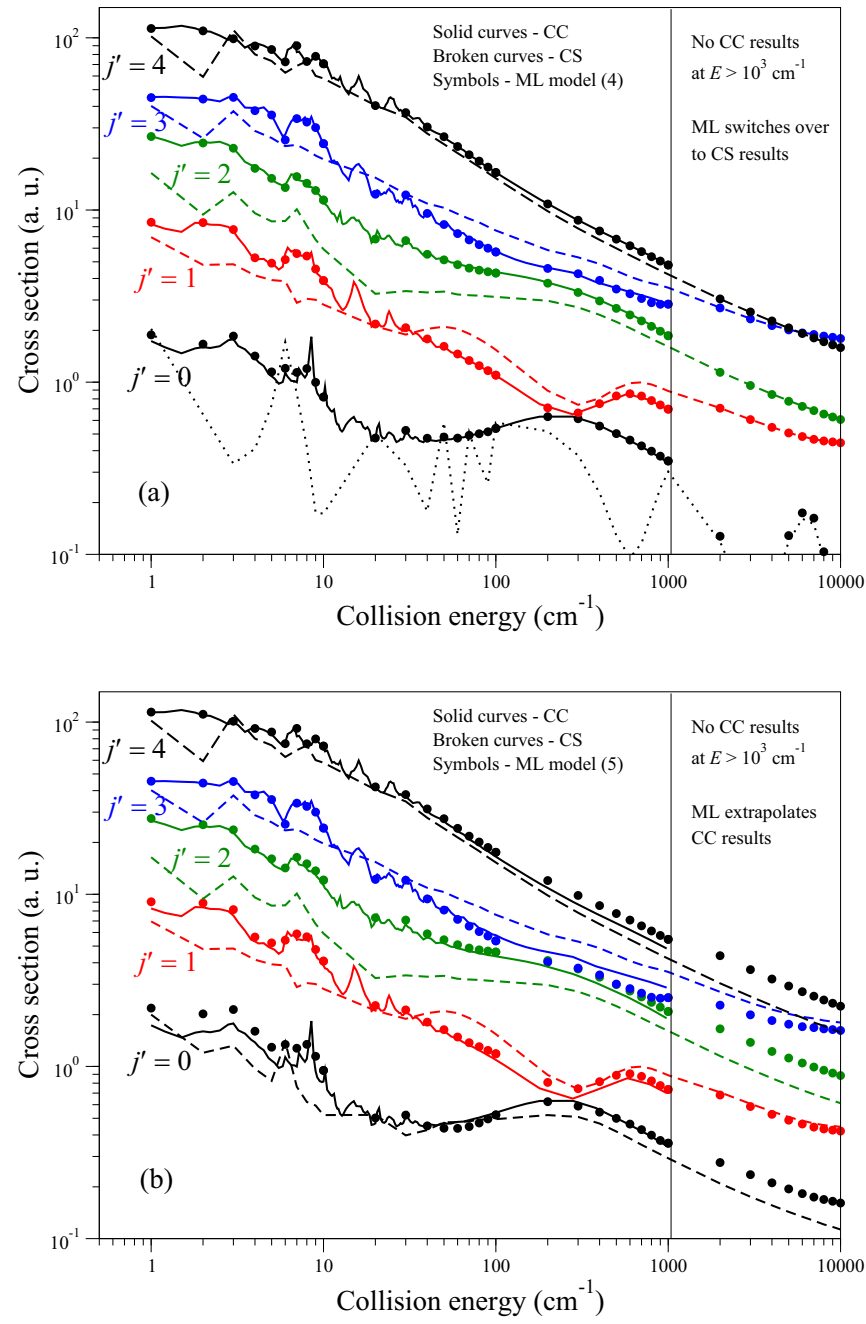

FIG. 3. Cross sections for purely rotational relaxation in collisions of vibrationally $\left(v_{1}=1\right)$ and rotationally $\left(j_{1}=5\right)$ excited $\mathrm{SiO}$ with $\mathrm{H}_{2}\left(v_{2}=0, j_{2}=0\right)$. The final rotational state of $\mathrm{SiO}$ is $j^{\prime}$. The solid curves are the $6 \mathrm{DCC}$ results, the dashed curves are the 5DCS results, and the symbols are the ML results. The dotted line in the upper panel represents the 5DCS results modulated by an arbitrary sinusoidal function. Upper panel: Algorithm (4) for the ML models. Lower panel: Algorithm (5). Note that the solid curves are not used for training the ML models. The ML models are trained using the 6DCC results only for the rotationally inelastic transitions of $\mathrm{SiO}$ in the ground vibrational state $v_{1}=0$. There are no $6 \mathrm{DCC}$ results to train the models at collision energies $>10^{3} \mathrm{~cm}^{-1}$.

approach analogous to $\Delta$ learning [33], with an independent GP introduced to describe the difference between rigorous and approximate results, or an approach using multioutput GPs designed to learn correlations both between inputs and between multiple outputs, simultaneously. We have shown that the method based on multioutput GPs has the potential to extrapolate accurate results to the range of Hamiltonian parameters, where rigorous calculations are unfeasible. The demonstrated transfer learning opens the possibility of making accurate predictions for quantum transitions that are out of reach of rigorous dynamics calculations.

Finally, we would like to comment on the stability of the ML predictions presented here. We have demonstrated 
that the ML predictions do not rely on the accuracy of the approximate calculations used for training the models. This is a consequence of the present formulation that aims to determine correlations between the approximate and rigorous results. The ML predictions depend on the number of rigorous calculations. However, because we are using the Bayesian approach, the ML predictions can be converged by gradually increasing the number of rigorous calculations. The final error of the ML predictions is limited by the generalization error but it must be smaller that the error of the approximate calculations, unless different transitions are completely uncorrelated. Correlations between different transitions, especially transitions with the same quantum number gaps, must be present, as long as the dynamics of different transitions are determined by the same potential energy surface.

Acknowledgment. This work was supported at Penn State by NSF Grant No. PHY-1806180, at UGA by NASA Grant No. NNX16AF09G, at UNLV by NSF Grant No. 1806334, and by NSERC of Canada.
[1] A. M. Arthurs and A. Dalgarno, The theory of scattering by a rigid rotator, Proc. R. Soc. London, Ser. A 256, 540 (1960).

[2] M. Berciu, Few-Particle Green's Functions for Strongly Correlated Systems on Infinite Lattices, Phys. Rev. Lett. 107, 246403 (2011).

[3] J. M. Hutson, Coupled channel methods for solving the boundstate Schrödinger equation, Comput. Phys. Commun. 84, 1 (1994).

[4] E. Roueff and F. Lique, Molecular excitation in the interstellar medium: Recent advances in collisional, radiative, and chemical processes, Chem. Rev. 113, 8906 (2019).

[5] W. E. Perreault, N. Mukherjee, and R. N. Zare, Quantum control of molecular collisions at 1 Kelvin, Science 358, 356 (2017).

[6] R. V. Krems, Cold controlled chemistry, Phys. Chem. Chem. Phys. 10, 4079 (2008).

[7] N. Balakrishnan, Perspective: Ultracold molecules and the dawn of cold controlled chemistry, J. Chem. Phys. 145, 150901 (2016).

[8] P. G. Jambrina, J. F. E. Croft, H. Guo, M. Brouard, N. Balakrishnan, and F. J. Aoiz, Stereodynamical Control of a Quantum Scattering Resonance in Cold Molecular Collisions, Phys. Rev. Lett. 123, 043401 (2019).

[9] R. V. Krems, Molecules in Electromagnetic Fields: From Ultracold Physics to Controlled Chemistry (Wiley, New York, 2018).

[10] G. Carleo and M. Troyer, Solving the quantum many-body problem with artificial neural networks, Science 355, 602 (2017).

[11] K. Ch'ng, J. Carrasquilla, R. G. Melko, and E. Khatami, Machine Learning Phases of Strongly Correlated Fermions, Phys. Rev. X 7, 031038 (2017).

[12] H. Saito, Solving the Bose-Hubbard model with machine learning, J. Phys. Soc. Jpn. 86, 093001 (2017).

[13] Y. Nomura, A. S. Darmawan, Y. Yamaji, and M. Imada, Restricted Boltzmann machine learning for solving strongly correlated quantum systems, Phys. Rev. B 96, 205152 (2017).

[14] O. A. von Lilienfeld, Quantum machine learning in chemical compound space, Angew. Chem., Int. Ed. 57, 4164 (2018).

[15] J. Han, L. Zhang, and E. Weinan, Solving many-electron Schrödinger equation using deep neural networks, J. Comput. Phys. 399, 108929 (2019).

[16] K. T. Schütt, M. Gastegger, A. Tkatchenko, K. R. Müller, and R. J. Maurer, Unifying machine learning and quantum chemistry with a deep neural network for molecular wavefunctions, Nat. Commun. 10, 5024 (2019).
[17] K. Choo, A. Mezzacapo, and G. Carleo, Fermionic neuralnetwork states for $a b$ initio electronic structure, Nat. Commun. 11, 2368 (2020)

[18] D. Luo and B. K. Clark, Backflow Transformations via Neural Networks for Quantum Many-Body Wave Functions, Phys. Rev. Lett. 122, 226401 (2019).

[19] J. Hermann, Z. Schätzle, and F. Noé, Deep neural network solution of the electronic Schrödinger equation, arXiv:1909.08423.

[20] F. Noé, S. Olsson, J. Köhler, and H. Wu, Boltzmann generators: Sampling equilibrium states of many-body systems with deep learning, Science 365, eaaw1147 (2019).

[21] D. Koner, O. T. Unke, K. Boe, R. J. Bemish, and M. Meuwly, Exhaustive state-to-state cross sections for reactive molecular collisions from importance sampling simulation and a neural network representation, J. Chem. Phys. 150, 211101 (2019).

[22] C. E. Rasmussen and C. K. I. Williams, Gaussian Processes for Machine Learning (The MIT Press, Cambridge, MA, 2006).

[23] D. K. Duvenaud, H. Nickisch, and C. E. Rasmussen, Additive Gaussian processes, in Advances in Neural Information Processing Systems 24, edited by J. Shawe-Taylor, R. S. Zemel, P. L. Bartlett, F. Pereira, and K. Q. Weinberger (Curran Associates, Red Hook, NY, 2011), p. 226.

[24] D. K. Duvenaud, J. Lloyd, R. Grosse, J. B. Tenenbaum, and Z. Ghahramani, Structure discovery in nonparametric regression through compositional kernel search, Proceedings of the 30th International Conference on Machine Learning Research 28 , 1166 (2013).

[25] R. Vargas-Hernandez, J. Sous, M. Berciu, and R. V. Krems, Extrapolating Quantum Observables with Machine Learning: Inferring Multiple Phase Transitions from Properties of a Single Phase, Phys. Rev. Lett. 121, 255702 (2018).

[26] A. Kamath, R. A. Vargas-Hernandez, R. V. Krems, T. Carrington, Jr., and S. Manzhos, Neural networks vs Gaussian process regression for representing potential energy surfaces: A comparative study of fit quality and vibrational spectrum accuracy, J. Chem. Phys. 148, 241702 (2018).

[27] R. Vargas-Hernandez, Y. Guan, D. H. Zhang, and R. V. Krems, Bayesian optimization for the inverse scattering problem in quantum reaction dynamics, New J. Phys. 21, 022001 (2019).

[28] R. V. Krems, Bayesian machine learning for quantum molecular dynamics, Phys. Chem. Chem. Phys. 21, 13392 (2019).

[29] M. C. Kennedy and A. O‘Hagan, Bayesian calibration of computer models, J. R. Stat. Soc. B 63, 425 (2001). 
[30] J. Cui and R. V. Krems, Gaussian Process Model for Collision Dynamics of Complex Molecules, Phys. Rev. Lett. 115, 073202 (2015).

[31] P. Houston, A. Nandi, and J. Bowman, A Machine Learning Approach for Prediction of Rate Constants, J. Phys. Chem. Lett. 10, 5250 (2019).

[32] A. E. Wiens, A. V. Copan, and H. F. Schaefer, Multi-Fidelity Gaussian Process Modeling for Chemical Energy Surfaces, Chem. Phys. Lett.: X 3, 100022 (2019).

[33] R. Ramakrishnan, P. O. Dral, M. Rupp, and O. A. von Lilienfeld, Big data meets quantum chemistry approximations: The $\Delta$-machine learning approach, J. Chem. Theory Comput. 11, 2087 (2015).

[34] E. Bonilla, K. M. Chai, and C. Williams, Multi-task Gaussian process prediction, in Advances in Neural Information Processing Systems 20, edited by J. C. Platt, D. Koller, Y. Singer, and S. T. Roweis (Curran Associates, Red Hook, NY, 2008), pp. $153-160$.

[35] See Supplemental Material at http://link.aps.org/supplemental/ 10.1103/PhysRevResearch.2.032051 for details of the ML models, including the illustration of convergence of the model predictions.
[36] B. H. Yang, P. Zhang, X. Wang, P. C. Stancil, J. M. Bowman, N. Balakrishnan, and R. C. Forrey, Quantum dynamics of $\mathrm{CO}-\mathrm{H}_{2}$ in full dimensionality, Nat. Commun. 6, 6629 (2015).

[37] B. H. Yang, N. Balakrishnan, P. Zhang, X. Wang, J. M. Bowman, R. C. Forrey, and P. C. Stancil, Full-dimensional quantum dynamics of $\mathrm{CO}$ in collision with $\mathrm{H}_{2}$, J. Chem. Phys. 145, 034308 (2016).

[38] B. H. Yang, P. Zhang, C. Qu, H. Wang, P. C. Stancil, J. M. Bowman, N. Balakrishnan, B. M. McLaughlin, and R. C. Forrey, Full-dimensional quantum dynamics of $\mathrm{SiO}$ in collision with $\mathrm{H}_{2}$, J. Phys. Chem. A 122, 1511 (2018).

[39] C. Castro, K. Doan, M. Klemka, R. C. Forrey, B. H. Yang, P. C. Stancil, and N. Balakrishnan, Inelastic cross sections and rate coefficients for collisions between $\mathrm{CO}$ and $\mathrm{H}_{2}$, Mol. Astrophys. 6, 47 (2017).

[40] B. H. Yang, P. C. Stancil, N. Balakrishnan, and R. C. Forrey, Rotational quenching of $\mathrm{CO}$ due to $\mathrm{H}_{2}$ collisions, Astrophys. J. 718, 1062 (2010).

[41] A. A. Radzig and B. M. Smirnov, in Reference Data on Atoms, Molecules, and Ions, Springer Series in Chemical Physics Vol. 31 (Springer, Berlin, 1985). 